\title{
NOTE ON QUASIGROUPS AND TREES
}

\author{
by I. M. H. ETHERINGTON \\ (Received 4th January 1963)
}

If letters $a, b, c, \ldots$ are used to denote points of a nondegenerate plane cubic curve, other than the singular point if any, and if the product $a b$ is defined as the third point of the curve collinear with $a$ and $b$, we obtain an algebraic system having nonassociative multiplication ( $a b . c \neq a . b c$ in general). It is in fact a totally symmetric entropic quasigroup (these terms are defined below). This idea, which was put forward at a meeting of the Edinburgh Mathematical Society a few years ago, will be exploited in a forthcoming paper. Such quasigroups have many properties which can be interpreted geometrically. Or, conversely, known properties of cubic curves suggest theorems about totally symmetric entropic quasigroups, which if established will involve a gain in generality, since not every such quasigroup can be "placed" on a cubic.

The purpose of the present note is to mention certain properties ("symmetries") possessed by any totally symmetric entropic quasigroup, which are most conveniently expressed generally in terms of trees. The relevance to geometry on the cubic may be indicated briefly by an example. Suppose that $a, b, c, d, e, f$ are the six points where some conic cuts the cubic. It can be shown that the condition for this may be written $a b . c d=e f$, or equally $(a b . c d) e=f$. Either of these must therefore be a relation which involves the six elements $a, b, c, d, e, f$ symmetrically. It follows also that $(a b . c d) e$ is a formula for the sixth point in which the conic through five given points of the cubic meets the cubic; it must therefore be a symmetric function of its five arguments. It is with such symmetries of equations and functions that this note is concerned, in a general totally symmetric entropic quasigroup. Postulates defining such a quasigroup will be introduced one at a time.

Since nonassociative multiplication is to be considered, to avoid writing many brackets, let it be understood that a dot separates factors whose multiplication is to be delayed, e.g. $(a b . c d) e . f g$ means $[\{(a b)(c d)\} e](f g)$. The word product is to be understood as meaning the product of a number of factors suitably bracketed (or perhaps a single element), and an equation means an equality between two products.

With any product we can associate a tree. The tree may be thought of as a pedigree in which the product is regarded as being descended from the partial products and ultimately from the separate factors (example: fig. 1). This is a tree in which one vertex is distinguished as the root of the tree, and proceeding from the root the tree bifurcates repeatedly. 
If two products are equated, let us represent this by drawing the two trees and joining their roots (example: fig. 2). We get thus a single tree of a different kind; it has the property that every vertex lies on either one or three edges, and now one edge is distinguished-let us call it the trunk of the tree-representing the equality sign.

Now suppose that we have a groupoid $G$ (i.e. a set closed under multiplication) which obeys the demisymmetric law (so called by A. Sade (5))

$$
a b . a=b .
$$

for all elements $a, b$ of $G$. Then $a b=c$ implies $c a=b$, and conversely, if this is so, then (1) holds. Then likewise $c a=b$ implies $b c=a$, which in turn implies $a b=c$. Thus (1) is equivalent to the statement that each of the equations

$$
a b=c, \quad c a=b, \quad b c=a
$$

implies the other two. Hence (1) is equivalent to the identity $b . a b=a$, as observed by Sade ((5), p. 91). We observe that $G$ is necessarily a quasigroup

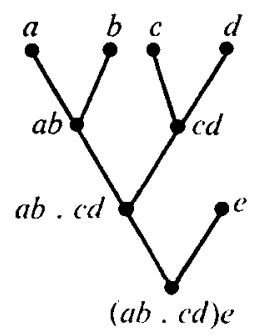

Fig. 1.

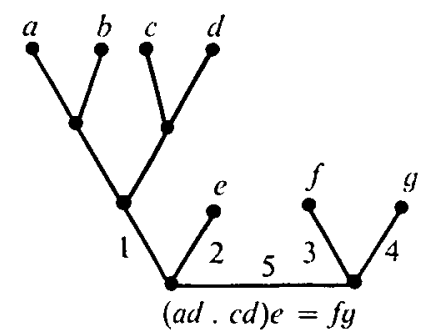

Frg. 2.

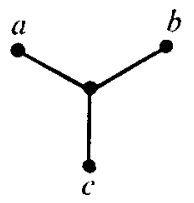

Fig. 3.

(i.e. a groupoid in which an equation $a b=c$ can always be solved uniquely for $a$ or $b$ given the other two).

Let us express this result in terms of trees. The tree for $a b=c$ is shown in fig. 3, $c$ being represented by the trivial tree which consists of a single vertex only; and the demisymmetric law asserts that it does not matter which edge we regard as the trunk.

This can be generalised. The equivalence of equations ( $1 a)$ shows that if the left side of an equation is a product of two factors we can transfer either factor to the other side of the equation, the left factor to the right of it or the right factor to the left of it. Also if the right side is a product (as in $c=a b$ ), the same statement applies. These four possibilities mean that in any tree representing a given equation, instead of the given trunk we can take any edge adjacent to it as trunk. Thus in fig. 2 , instead of the given trunk 5 , we could take $1,2,3$ or 4 as trunk and read the tree as asserting respectively

$$
a b . c d=e . f g, \quad e=f g .(a b . c d), \quad g .(a b . c d) e=f, \quad(a b . c d) e . f=g .
$$

Since this can be applied repeatedly we have the following result:

If the demisymmetric law holds, then in any tree representing an equation ANY edge can be taken as the trunk. 
(Of course it may be convenient to redraw the tree to facilitate rereading it, placing the trunk horizontal and the other branches above it.) From this point of view (1) and $b . a b=a$ are rereadings of the tree for $a b=a b$.

Now let us impose on $G$ two further postulates: firstly the commutative law

$$
a b=b a
$$

then the quasigroup $G$ becomes totally symmetric $(2,6)$, i.e. an equation $a b=c$ implies all six equations derived by permuting $a, b, c$; and finally the entropic law

$$
a b . c d=a c . b d \text {. }
$$

Quasigroups with this property were originally called abelian by Murdoch $(3,4)$ and Bruck (2). They are a generalisation of abelian groups, and are also isotopic to abelian groups (2). However, abelian is elsewhere understood as meaning "commutative". Etymologically entropic refers to "inner turning", meaning that the two inner factors in $a b . c d$ can be exchanged. Other names for the property (3) have been proposed, e.g. bisymmetric (Aczél) and medial (Stein).

(2) and (3) imply that $a b . c d$ is a symmetric function (equal to each expression obtainable by permuting the letters). So is $(a b . c d)(e f . g h)$, since we can interchange $c d$ with $e f$ and also apply the entropic law within each parenthesis. In fact we have a sequence of symmetric functions

$$
a b, \quad a b . c d,(a b . c d)(e f . g h), \quad \ldots
$$

(as noted by Aczél (1)). If here any of the partial products are replaced by single factors we obtain a function which is symmetric in the unaffected factors. For example, $(a b . c)(e . g h)$ is symmetric in $a, b, g, h$ (for in a quasigroup $c$ can always be represented as $c_{1} d$ and $e$ as $e_{1} f$ ); and this expression, since it is of the form $\alpha c . e \beta$, is also symmetric in $c$ and $e$.

Expressed in terms of trees the argument shows that in a commutative entropic quasigroup any product is symmetric in all those of its factors which appear at the same altitude in the tree.

In this statement the altitude of a vertex in a rooted tree is the number of edges which separate that vertex from the root; the altitude of the tree itself is the greatest of the altitudes of its vertices. Thus in the tree for $(a b . c)(e . g h)$, the factors $a, b, g, h$ are at altitude 3 , while $c, e$ are at altitude 2 ; the altitude of the tree is 3 .

If (1) also holds we can prove a result stronger than (4). I am indebted to I. R. Porteous for pointing out the algebraic result which I now express in terms of trees.

A factor $a$ can always be replaced by $x a, x$ and thus have its altitude raised by 2 . Hence in a totally symmetric entropic quasigroup any product is symmetric in all those of its factors which are at even altitude in the tree, and is also symmetric in all the rest (those at odd altitude).

E.M.S.-P 
Another way of expressing this result is the following. In a totally symmetric entropic quasigroup any two factors of a product can be interchanged if in the tree which represents the product they are separated by an even number of edges. In this form the same result applies to equations; for when the equation is represented by a tree we can always pick as trunk an edge which will put the two factors in question on the same side of the equation.

The final conclusion which we have reached is that in a totally symmetric entropic quasigroup, in any product or equation, if we divide the factors involved into two sets, each set corresponding to vertices in the tree separated by even numbers of edges, then the product or equation is symmetric in each set. For example, the equation $(a b . c d) . e f=g h . i$ expresses a relation which is symmetric in $a, b, c, d, \dot{g}, h$, and also in $e, f, i$; we can also regard $g h$ as a single element and interchange it in the equation with $e$ or $f$, and other such exchanges are possible, as will be seen on drawing the tree.

\section{REFERENCES}

(1) J. Aczél, On mean values, Bull. Amer. Math. Soc., 54 (1948), 392-400.

(2) R. H. BRUCK, Some results in the theory of quasigroups, Trans. Amer. Math. Soc., 55 (1944), 19-52.

(3) D. C. Murdoch, Quasi-groups which satisfy certain generalized associative laws, Amer. J. Math., 61 (1939), 509-522.

(4) D. C. Murdoch, Structure of abelian quasi-groups, Trans. Amer. Math. Soc., 49 (1941), 392-409.

(5) A. SADE, Quasigroupes parastrophiques. Expressions et identités, Math. Nachr., 20 (1959), 73-106.

(6) S. K. Stein, On the foundations of quasigroups, Trans. Amer. Math. Soc., 85 (1957), 228-256.

\section{Mathematical Institute \\ THE UNIVERSITY \\ EDINBURGH}

